\title{
Laminar Constructions and Reciprocal Structures
}

\author{
Javier Barrallo ${ }^{1}$ (I) Francisco González-Quintial $^{1}$. \\ Antonio Sánchez-Parandiet ${ }^{1}$
}

\begin{abstract}
Reciprocal structures are constructed by using a wide variety of patterns. These designs are a good source of inspiration when working with laminar constructions (sheets). Using the same formal schemes, laminar constructions feature better bending of their elements along the shape of the model and the application of extra pressure on the connection joints. Many of the geometric constructions made at the University of the Basque Country and presented in this paper show structural, constructive and formal improvements in many reciprocal structures assembled using sheets instead of linear elements.
\end{abstract}

Keywords Reciprocal frame structures - Leonardo grids - Laminar constructions $\cdot$ Complex shapes $\cdot$ Developable surfaces

\section{Reciprocal Structures}

Although reciprocal structures have probably been known since antiquity, the first detailed sketches are found in the folio 899v of Leonardo da Vinci's Codex Atlanticus (Williams 2008). In recent years the scientific community has begun to consider these structures as something more than a historical curiosity. Some scholars attribute to Villard de Honnecourt the first drawing of a reciprocal

Javier Barrallo

javier@barrallo.com

Francisco González-Quintial

0.pgquintial@gmail.com

Antonio Sánchez-Parandiet

antonio.sanchez@ehu.es

1 E.T.S Arquitectura, UPV-EHU The University of the Basque Country, Plaza Oñati 2, 20018

Donostia-San Sebastián, Spain 
structure, found in the sketchbook he compiled between 1225 and 1250, but his sketch is imprecise and in any case, it should be considered more as a constructive detail than a real structure (see Duvernoy 2008).

The principle of structural reciprocity is the use of elements that lean on each other mutually in a three-dimensional structure (Pugnale et al. 2011). A minimum of three elements is needed, and some friction amongst them is required. Ideally, the unions of elements have no mechanical connections, just pressure and friction, but it is not unusual to find in these constructions notches, ropes, wires or rivets to secure the structure at the contact points (Popovic 2007).

Reciprocal structures have been studied in depth for over 20 years by the Dutch sculptor Rinus Roelofs, who has constructed several impressive wood domes. This type of construction received the name of "Leonardo Grids" because they used the same constructive principles detailed by Leonardo da Vinci. Rinus Roelofs expanded extensively the sketches of Leonardo into a complex constructive system featuring many different patterns, changes of curvatures and increasing the number of layers (Fig. 1). These techniques were profusely tested during the two seminars organized by Nexus in Óbidos, Italy, in 2003 (Duvernoy 2008) and in Perdizes, Portugal, in 2013 (Williams and Xavier 2014).

\section{From Beams to Planar Elements}

In 1998 Rinus Roelofs presented in Escher Centennial Congress at Rome a work entitled "Not the Tiles but the Joints" showing many illustrations featuring the joints from the tiles that M. C. Escher used in his artwork. The connection of Escher together with the discovery of Leonardo grids inspired Rinus Roelofs to construct an amazing set of well-known reciprocal frame structures (Roelofs 2008). Curiously, the later works of Rinus Roelofs and other scholars were mainly based on beam elements, forgetting the spaces between beams as a geometric basis for new constructions.

(a)

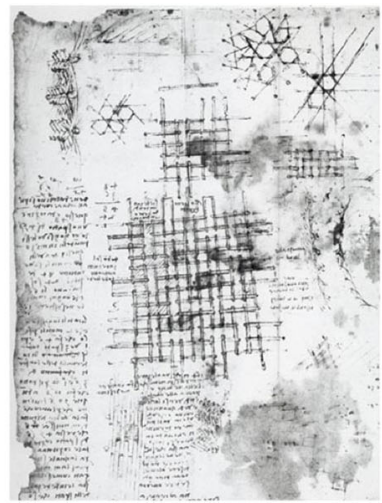

(b)

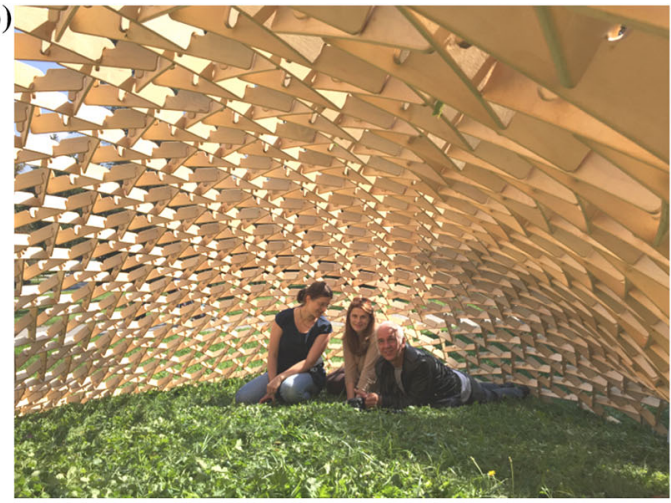

Fig. 1 Sculptor Rinus Roelofs inside one of his domes based on Leonardo sketches. Photo: authors 
The first part of our research consisted on the study of planar elements designed as closing surfaces interlaced with linear reciprocal structures. Reciprocal structures have been widely studied previously as mere structural experiments but it is obvious that many architectural applications need planar closing elements to become functional.

The enclosure of a linear reciprocal structure is always difficult mainly due to their spatial interlocked parts and their protruding ends. Our first experiments consisted on the closing of the complete surface over the reciprocal structure using plates as shown in Fig. 2.

The cutting of each piece responds to the geometry of the pattern with a slight modification of its vertices and edges. The latches from the laminar pieces tie the rods forming a more compact package and preventing from collapse. The same technique works with many other patterns of reciprocal structures, as it is a clear and well-defined parametric generation process. The plates work as a very efficient structural bracing system, at least with the scaled prototypes we have worked with.

This research is more a geometric than a constructive study, so the aspects related to the watertight envelope is not really considered as a priority problem. The line of research focuses on the possibilities that the sheets can offer following geometric patterns from the organizations of reciprocal structures. Further, this analysis initiates the exploration of the possibilities that can offer the use of sheets without the bar linear elements.

\section{Passive and Active Bending}

Reciprocal structures formed by the arrangement of rigid linear elements may become stable simply by the friction generated in the joints between bars. In these cases, the weight of the bars generates the necessary friction to balance the structure.

However, if we work with bars flexible enough, the structure is allowed to change its curvature depending on the bending capability of the elements (Fig. 3a). The new
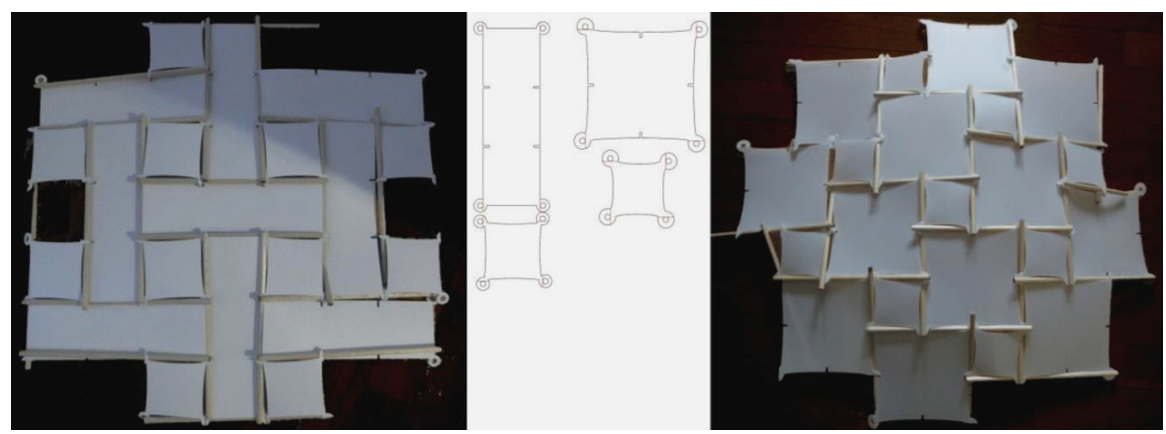

Fig. 2 Two basic structural frames with its laminar closing. The one on the left is based on planar elements $3 \times 1$ and $1 \times 1$, the one on the right has tiles $1 \times 1$ and $2 \times 2$. Images: authors 
(a)

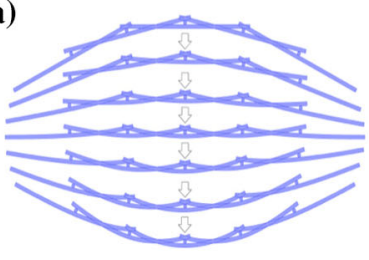

(b)

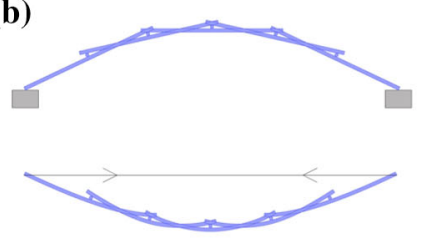

(c)

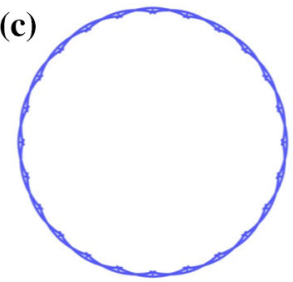

(d)

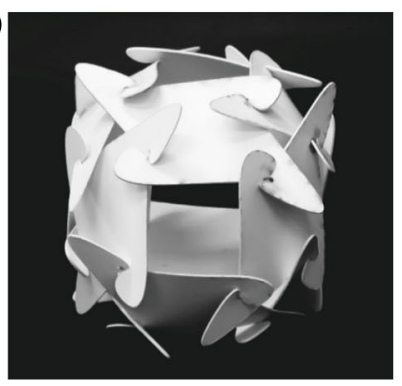

(e)

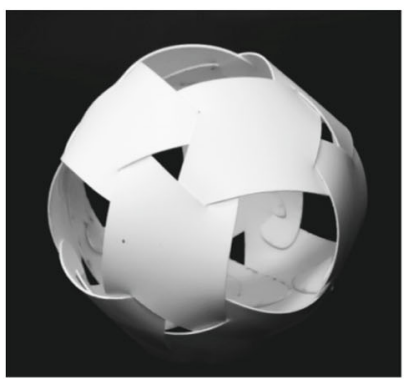

Fig. 3 a Changes of curvature in a reciprocal structure; $\mathbf{b}$ structure under passive and active bending (with a tensor); c closed structure (no tensor needed); $\mathbf{d}$ polyhedron with outwards flaps under passive bending; e same polyhedron with same pieces but inward flaps under active bending

structure may be balanced under the action of a tensor (Fig. 3b) or create a closed structure linking the bars if the model bends until the ends meet (Fig. 3c).

If we substitute the bending passive rods by laminar elements, these are allowed to be folded in the direction of the axis of weakest inertia and the set turns to behave as a structure with bending active planar patches. Active bending increases the friction forces generated by the weight of the elements to the normal forces to the surface, tending to stabilize the ensemble.

As a consequence, the interaction amongst the laminar elements of the reciprocal structure implies a difference in the geometry of the resulting structure. The pieces are curved following a continuous surface. This difference can be seen in the organization of the same elements depending on the position of the connecting flaps inwards or outwards, resulting on a passive or active bending structure (Fig. 3d, e).

\section{Laminar Constructions Without Linear Elements}

In spite of the increase of experiments with on reciprocal structures in recent years, the scarcity of references to constructions with laminar elements is striking. Some isolated cases seem to appear more by chance than by any systematic analysis with different geometries and settings. There also exist very interesting exceptions, such as the works of Baverel and Pugnale (2014) and others (Kohlhammer and Kotnik 2011; Parigi and Pugnale 2014; Sánchez et al. 2010).

In most cases the planar elements are introduced in the reciprocal structures by expanding the linear beam elements vertically. In this way, the length of these new 
elements allows the introduction of deep interlocked joints to fix the structure in a very similar way to that used in the London Serpentine Pavilion by Siza et al. (2005).

By increasing the thickness of these pieces and the angle of the joints, very solid structures may be created. But far from being flat structures composed of planar elements, they are rather spatial structures, composed of sheets assembled in different planes following the scheme of an ordinary linear reciprocal structure.

One of the most interesting results of our work shows that the sheets covering the empty spaces of a linear reciprocal structure work perfectly as substitutes of the structural elements (rods). The sheets both support the construction and act as a planar closing surface without the presence of any beam element.

An example is shown in Fig. 2a, which was assembled by associating pairs of rods and rectangular sheets. Each pattern admits several solutions for shapes and unions, but in general we perceive it as interlaced ribbons along orthogonal lines. Mechanical joints are not strictly necessary if there is enough friction amongst the pieces (Fig. 4).

Another model, Fig. 5a, represents a reciprocal pattern with square pieces. The borders of the squares work like the rods, flexing due to the thrust over the edges of the adjacent pieces. The original shape is distorted because of the presence of four tabs around the square. Two of the tabs lap over two sheets, while the other two give support to another pair of sheets. The upper and lower view of this structure can be seen in pictures Fig. 5b, c.

The blue arrows in Fig. 5d show the direction of the forces that the four white sheets apply over the blue one, alternating up and down directions depending on the situation of the tabs. Note that the eight tabs are concealed in the upper view and are visible in the lower view.

If the material of the sheets provides enough friction, the reciprocal structure will be stable; otherwise the use of tacks or glue is necessary. For many of the small scaled models developed in this paper we have used $0.8 \mathrm{~mm}$ polypropylene. Polypropylene is the lightest of commercial plastics and features a hinge effect that allows bending without breaking, unlike other similar plastics like PVC. Unfortunately it is very slippery (less friction), but it has a high resistance to many chemical products. Polypropylene produces a straight apparent contour when bending (see the

(a)

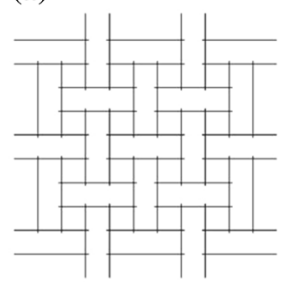

(b)

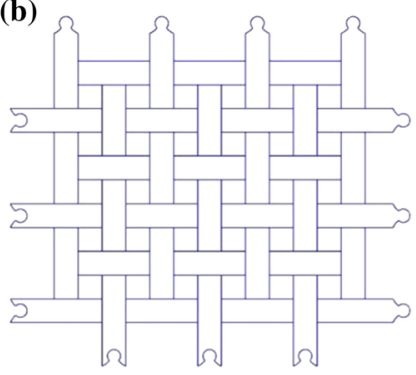

(c)

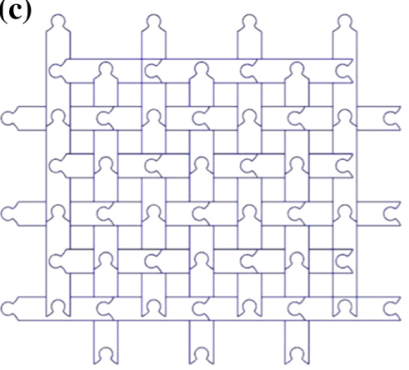

Fig. 4 a Reciprocal frame structure pattern and its translation into sheets, b upper view, c lower view. Images: authors 
(a)

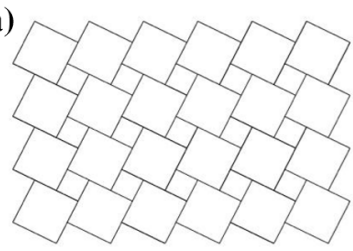

(b)

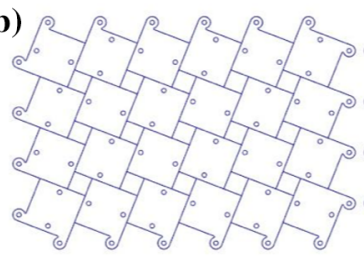

(c)

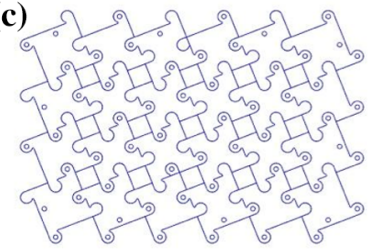

(d)

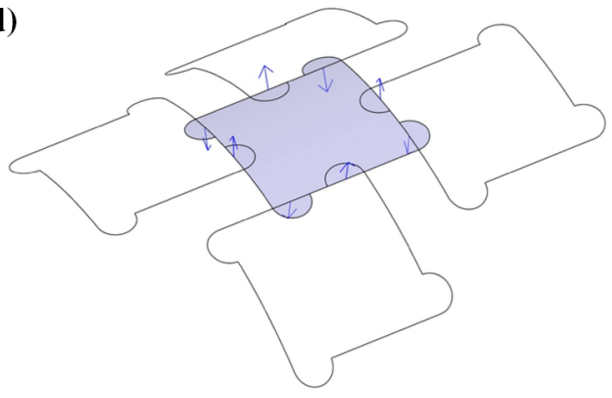

(e)

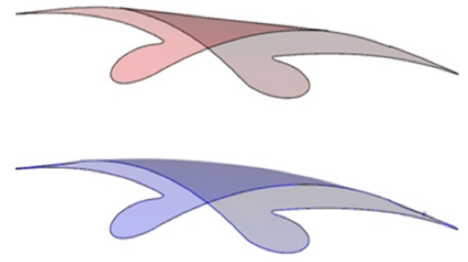

Fig. 5 a Reciprocal frame structure pattern with square sheets and without rods; $\mathbf{b}$ upper view; $\mathbf{c}$ lower view; $\mathbf{d}$ direction of the forces from the white sheets over the blue one; $\mathbf{e}$ apparent contours of one sheetred (above), straight; and blue (below), curved — due to the use of different materials. Images: authors (color figure online)

top image in Fig. 5e) meanwhile other materials like plastic foams produce more curved apparent contours (see the bottom image in Fig. 5e).

\section{Models Associated to Polygonal and Textile Patterns}

Up to this point, our work has focused on the fact that the sheets can cover the reciprocal frame structure working as substitutes of the linear structural elements. Hence no kind of bar, rod or beam is required, as sheets function simultaneously as structure and enclosure.

We have selected two different groups of geometries, which we call polygonal and textile patterns. In the first group, the polygonal patterns, the linear structure defines several types of polygons and we select those whose sides are in contact with each other. Considering the examples shown in Fig. 6, in the first pattern from hexagons and rhombi, rhombi are selected; in the second pattern, from triangles and hexagons, triangles are selected; in the third pattern from $1 \times 1$ squares and $2 \times 2$ squares, the larger ones are selected.

In order to join the sheets together it is necessary to add some tabs that work as joints between them. The bending of the sheets produces the necessary amount of force to fix the tabs to one another. On every par of tabs, one laps over the sheet, while the other gives support to another, generating a reciprocal system, as shown in Fig. 5d.When the material provides enough friction, the reciprocal frame structure will be stable; otherwise the use of a mechanical joint system (tacks or glue) will be necessary. 
(a)
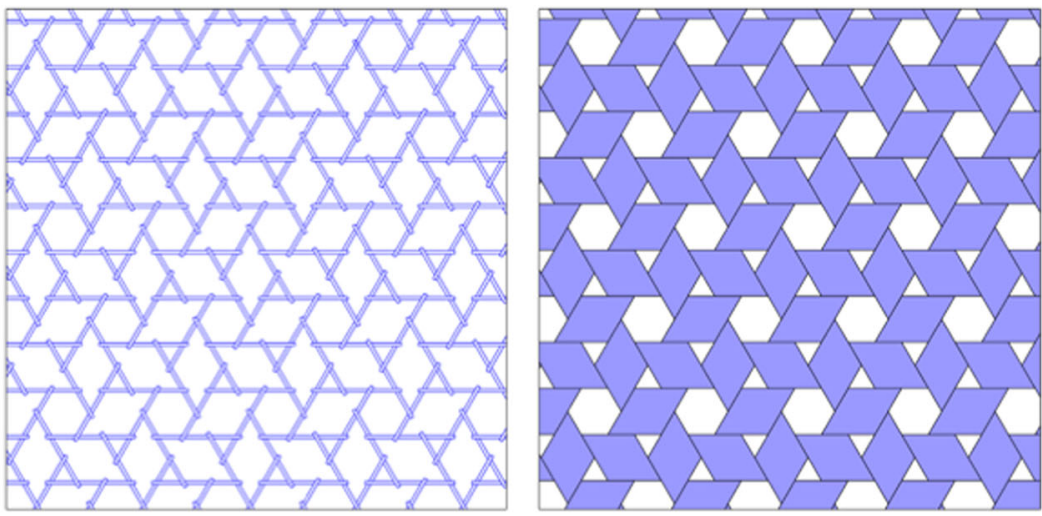

(b)
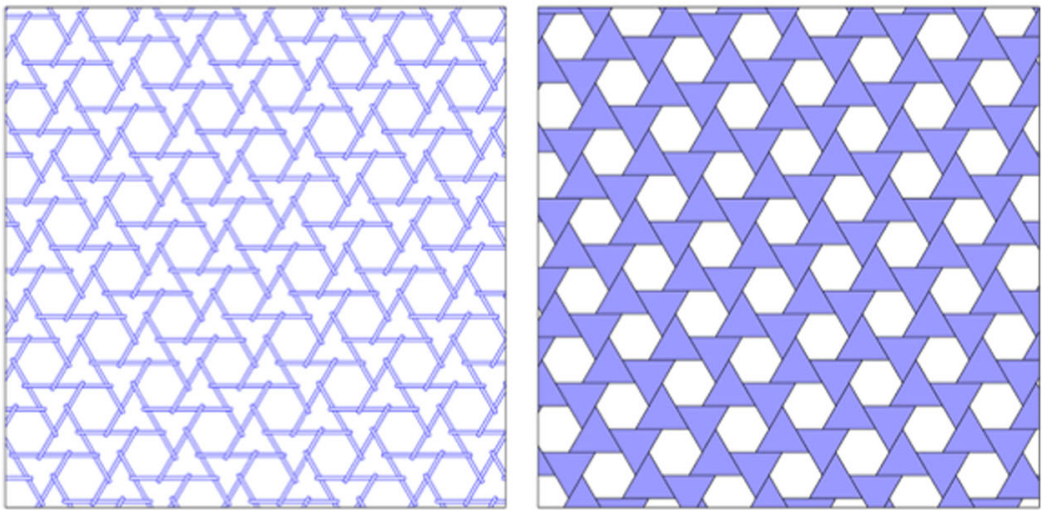

(c)
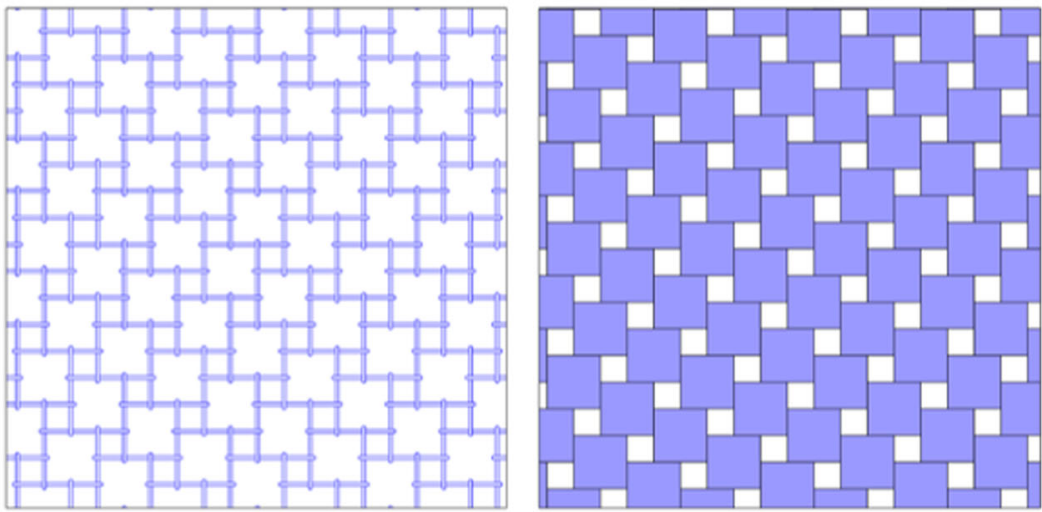

Fig. 6 Reciprocal models associated to polygons: a rhombi, b triangle, $\mathbf{c}$ square. Images: authors

The second group, named textile reciprocal patterns, follows the same process The shape is filled up using sheets as strips following a nesting or weaving system. The number of solutions to cover a surface using textile reciprocal patterns is really 
huge, depending on the kind of pattern we choose (Baverel and Popovic 2011) (Fig. 7).

\section{Comprehensive Analysis of Constructions Based on a Single Model of Sheet}

In order to study the possibilities of the laminar constructions described in previous sections we want to show some works developed with a unique model of sheet. All the constructions are based on laminar reciprocal structures working with flexion.

The selected piece is shaped rhombus based on two equilateral triangles with four tabs, two each of two slightly different shapes. Each tab occupies a quarter of the side of the diamond. The size of the tab is important because bigger tabs will produce tighter connections with more flexion while smaller tabs will leave more empty spaces in the model. If the tab occupies half of the side of the rhombus no empty spaces will be produced in the connections.

The research really started as a challenging game with the following initial proposal: how many geometric shapes might be possible to generate by using only a simple, rhombus-shaped piece of plastic. We used a laser-cutting machine and polypropylene sheets to cut several pieces. Then the construction challenge started and we discovered many geometric constructions and different combinations in the assembly of the sheets for the same geometrical construction.

The possibilities of assembling depend on the design of the piece and its capabilities to be fitted with identical ones. In some cases, slight alterations in the geometry of the sheet or the tab decreased the difficulties during assembling, but the goal of our challenge was the use of a unique geometry.

The following sections will show the amazing possibilities of this kind of constructions using a single tiling unit within a reciprocal frame structure (Fig. 8).

\section{Planar Surfaces and Cylinders}

The first logical generation is the construction of a planar surface that may be used as a guide to construct more sophisticated geometries. In this section we will show the planar constructions and the cylinders associated to them.

From our experiments we have succeeded in generating twelve planar patterns, all related to square or hexagonal grids. However, one of them is non-periodic and cannot be transformed into a cylinder because the geometry of the starting line of the plane cannot be connected to the opposite side after rolling the plane. Therefore, only eleven regular patterns may be converted into cylinders (Fig. 9).

Obviously, by extending the planar surface along the $y$-axis direction we extend the length of the cylinder and by extending it along the $x$-axis we generate cylinders with longer radii. Note that in some cases the joint of the cylinder may be displaced vertically producing different cylinders, although the pattern will be the same. 
(a)
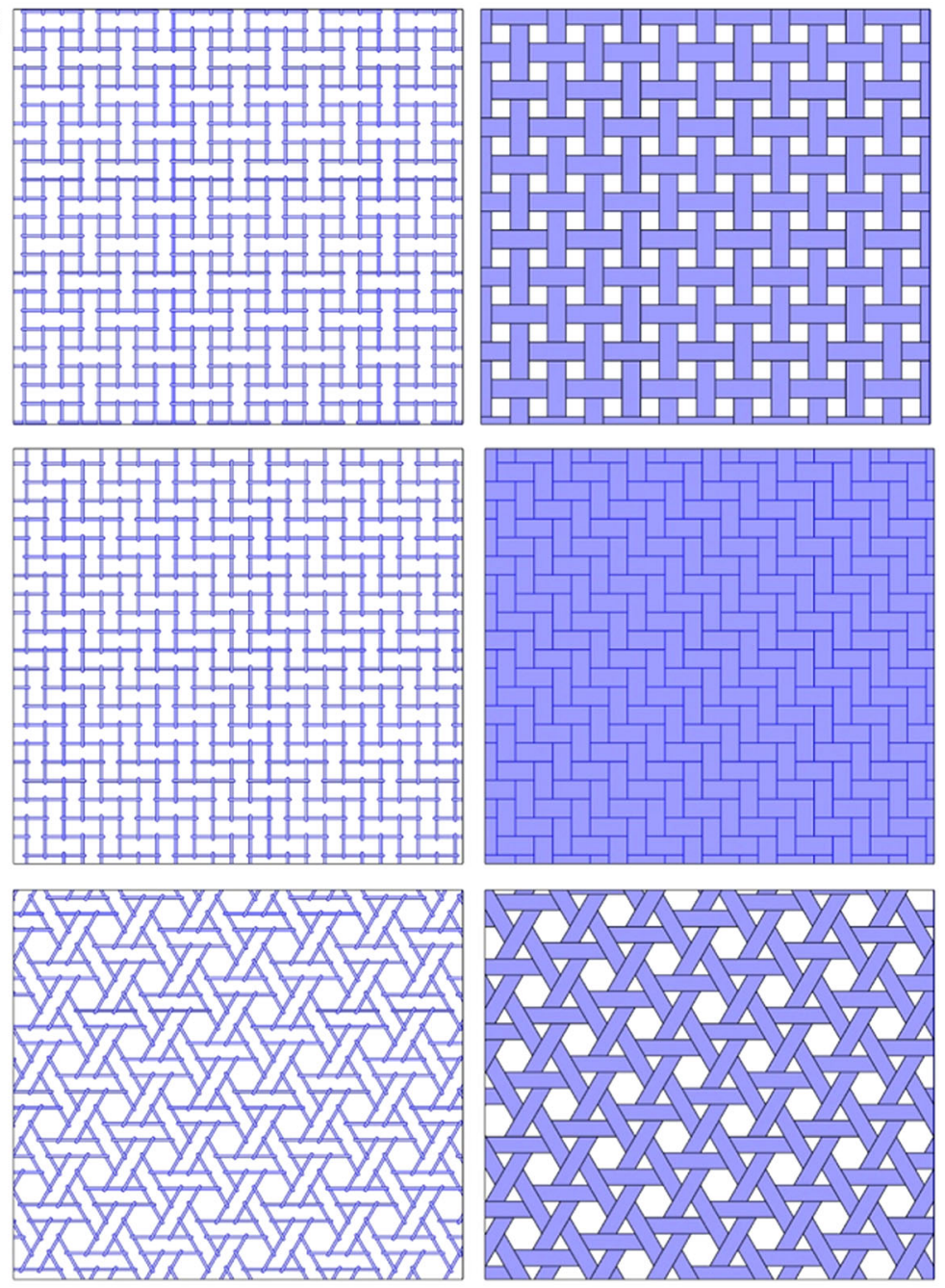

(b)

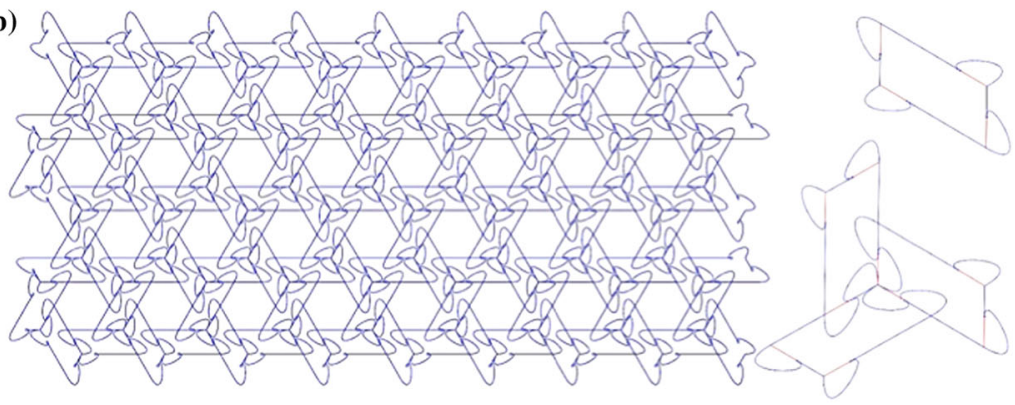

Fig. 7 a Above examples of three reciprocal models associated to textile patterns; $\mathbf{b}$ below the construction of the third textile pattern shown in detail. Images: authors 


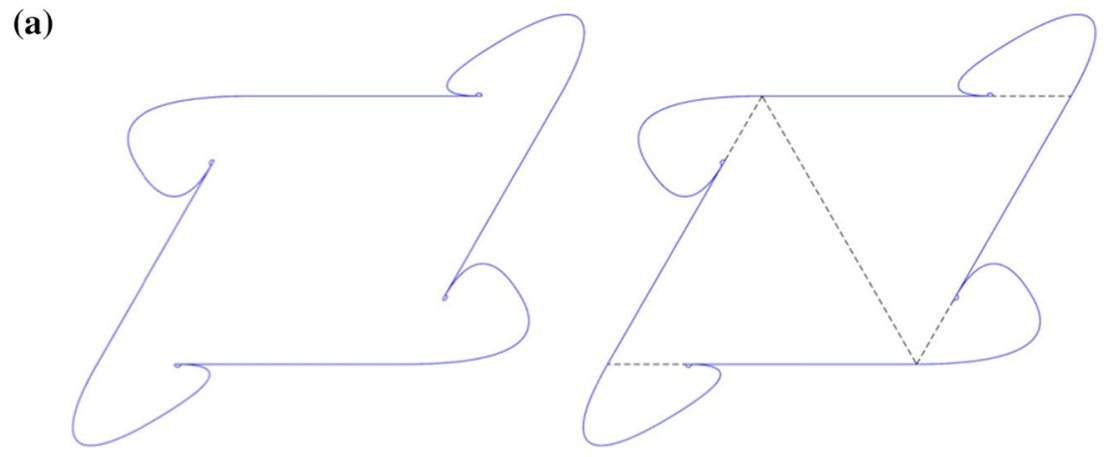

(b)

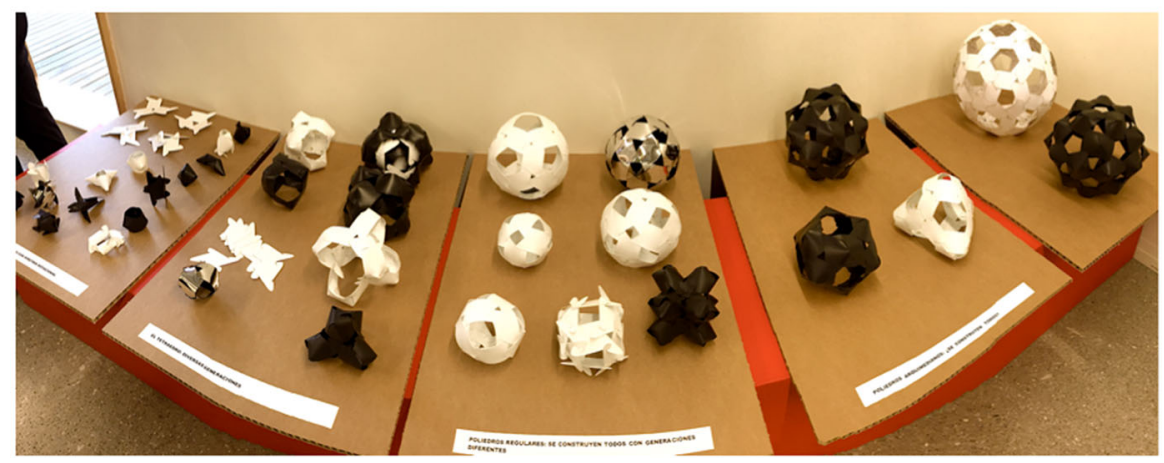

Fig. 8 a Detail of the selected model of sheet based on two equilateral triangles and four tabs in rotation. b Several polyhedra constructed using only the sheet shown above, as presented at Nexus Conference in Donostia-San Sebastián in June 2016. Images: authors

\section{Conical Shapes}

Just as a planar pattern can be bent into a cylinder, a pattern constructed with the shape of a circular sector may be warped into a conical shape. However, it is not easy to assemble both straight lines of the circular sector because they will fit correctly only when selected angles are used.

We have found several planar patterns which can be transformed into conical shapes (Fig. 10). We illustrate in this paper only four patterns, producing a total of fourteen cones depending on the angle used in the circular sector. Figure 10a shows a cone based on a circular sector of $180^{\circ}$; Fig. $10 \mathrm{~b}$ generates three different cones with angles of $90^{\circ}, 180^{\circ}$ and $270^{\circ}$; Fig. $10 \mathrm{c}$, d generate five cones each with angles in the circular sector of $60^{\circ}, 120^{\circ}, 180^{\circ}, 240^{\circ}$ and $300^{\circ}$.

Note that in some cases, when the apex of the cone is slimmer than the bending allowed by the sheet, the vertex may not be completed, creating a kind of oculus. 

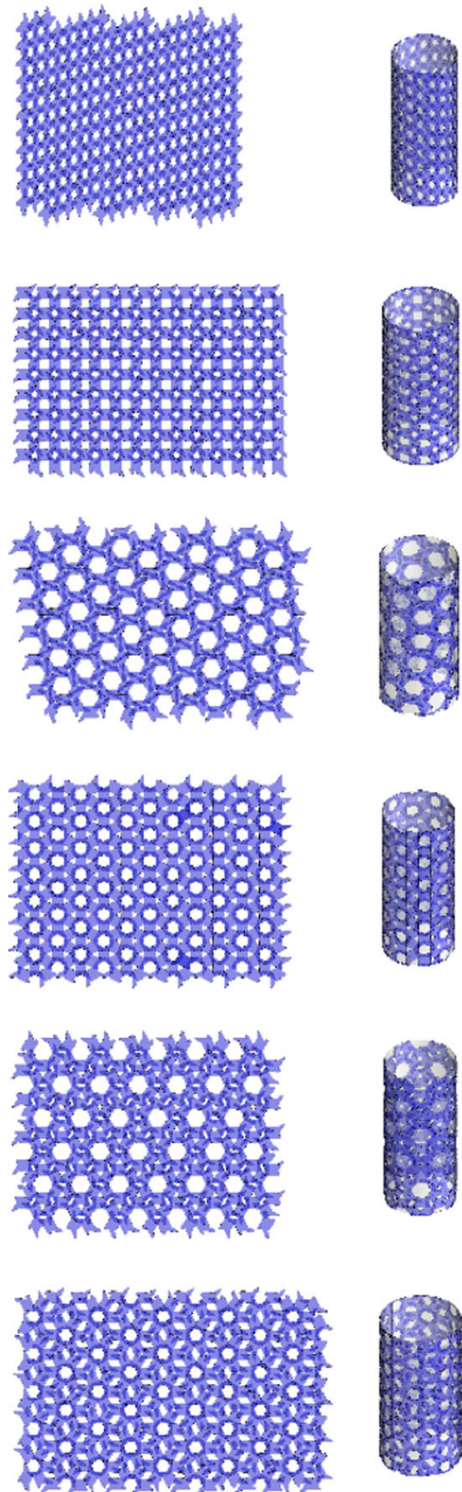
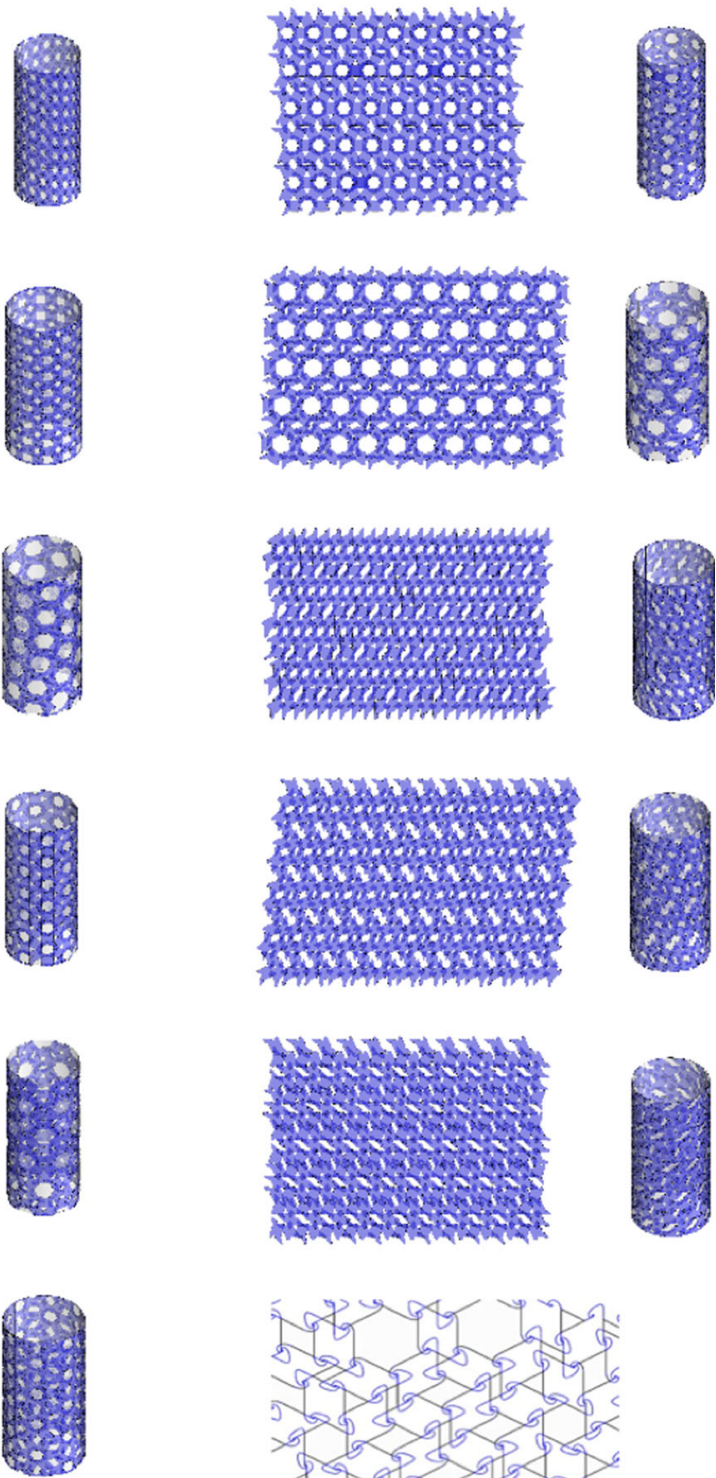

Fig. 9 The eleven planar patterns and the regular cylinders associated to them using only the sheet shown in Fig. 8a. There exists a twelfth non-periodical pattern that cannot be assembled into a cylinder. Images: authors

\section{Platonic and Archimedean Solids}

Polyhedra are always a good test for three-dimensional constructions. In this case, by weaving our sample piece, we can produce easily all the Platonic and Archimedean solids, that is, all regular and semi-regular convex polyhedra. 
(a)
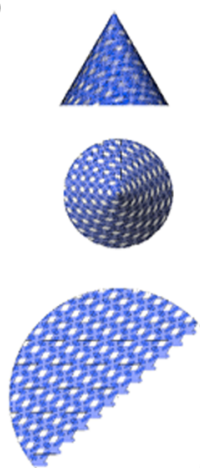

(b)
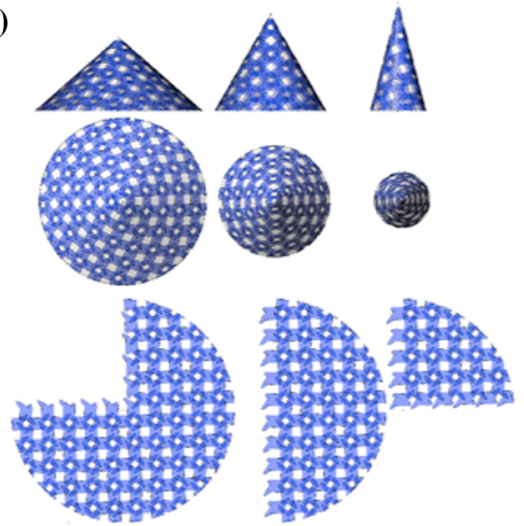

(c)

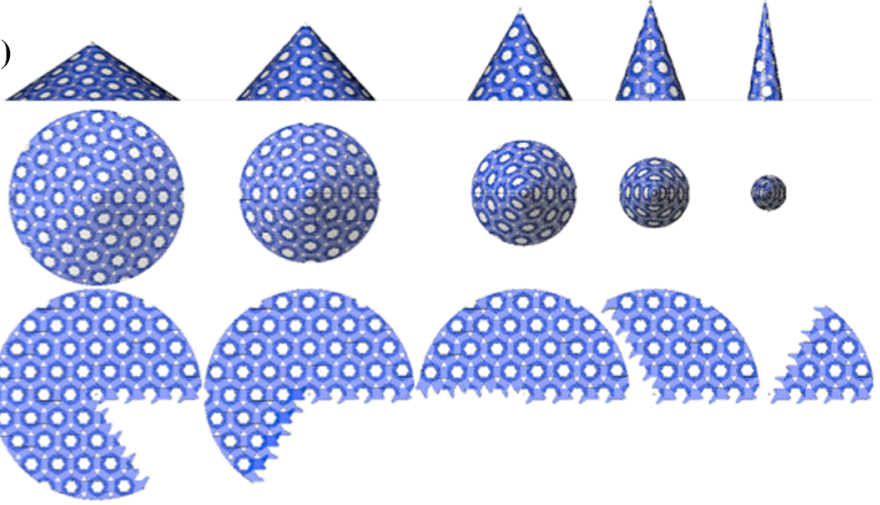

(d)
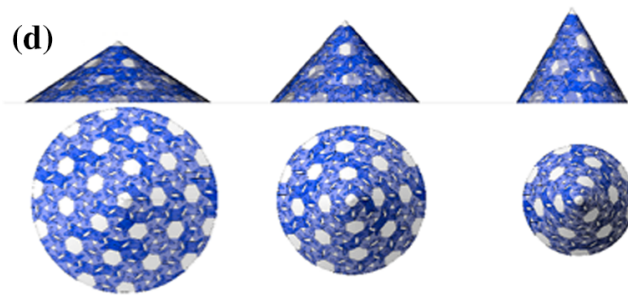

A

A
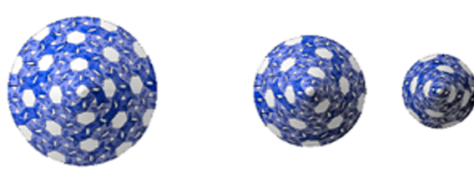

(2)
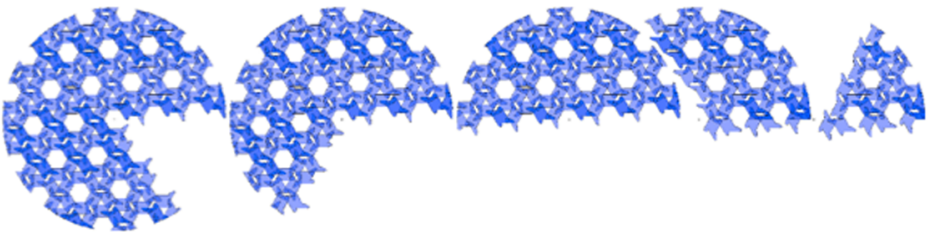

Fig. 10 Fourteen conical shapes may be developed from four different planar patterns $(\mathbf{a}, \mathbf{b}, \mathbf{c}, \mathbf{d})$ using only the sheet shown in Fig. 8a. Images: authors 
Every polyhedron has several associated laminar reciprocal patterns, so they can be constructed in different ways, depending in how pieces are assembled. The constructive material used is smooth and bends nicely so the pieces do not need any closing system apart from the bending-frictional mechanism.

Figure 11 shows the five regular polyhedra constructed in plastic, metal and wood. Models from Fig. 11a-e were constructed in small/medium size. The large icosahedron in Fig. 11f was constructed using medium density fibreboard and has a height of 150 centimetres. Although it is stable and self-supporting, all the laminar panels were secured with rivets as a precaution.

Note that all the polyhedra constructed in this section have two different shapes of perforations. One is the perforation corresponding to the regular polygon that generates the polyhedra (one type of polygon for regular polyhedra and two or three polygons for semi-regular polyhedra). The second perforation corresponds to the gaps in the union of the tabs, which leaves empty spaces with the polygonal shapes corresponding to the dual polyhedron. The rhomboid pieces are the edges; the tabs situated in the shorter sides define the apex of the polyhedron. The apexes generate empty spaces that cannot be considered faces; the empty spaces around the edges are the faces of the polyhedron.

Thus, the cube in 11a has six squares as generative polygons for a cube plus six triangles as gaps in the union of the tabs. The octahedron in Fig. 11c (dual of the cube) has six triangles as generative polygons and six squares in the joints. Dodecahedra (Fig. 11d), composed of twelve pentagons and Icosahedra (Fig. 11e), dual of dodecahedra and composed of twenty triangles, also present this duality in the number of polygons and the number of gaps created in the assembly of the tabs.

(a)

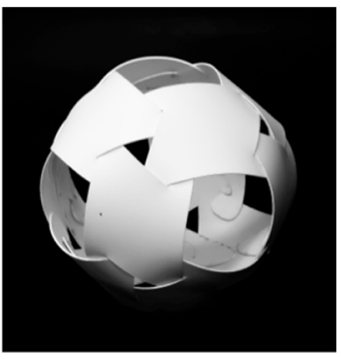

(d)

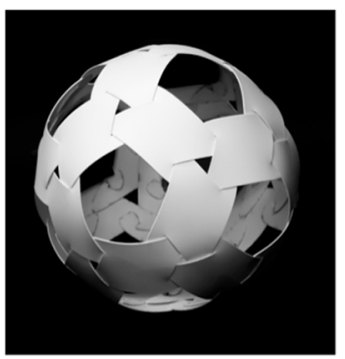

(b)

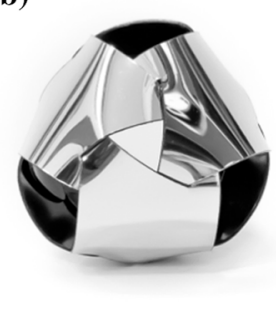

(e)

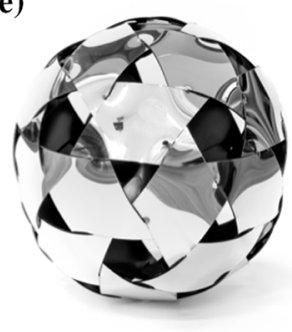

(c)

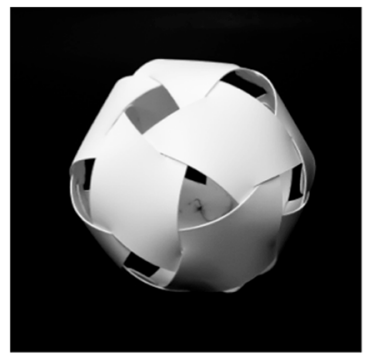

(f)

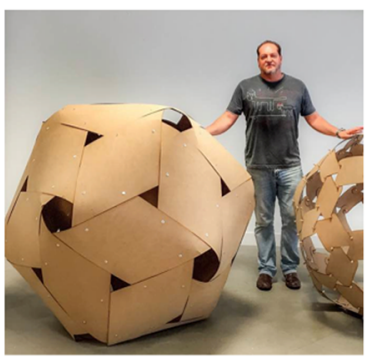

Fig. 11 Platonic solids: a cube; $\mathbf{b}$ tetrahedron; $\mathbf{c}$ octahedron; $\mathbf{d}$ dodecahedron; $\mathbf{e}$ icosahedron; $\mathbf{f}$ big scale icosahedron. Images: authors 
(a)

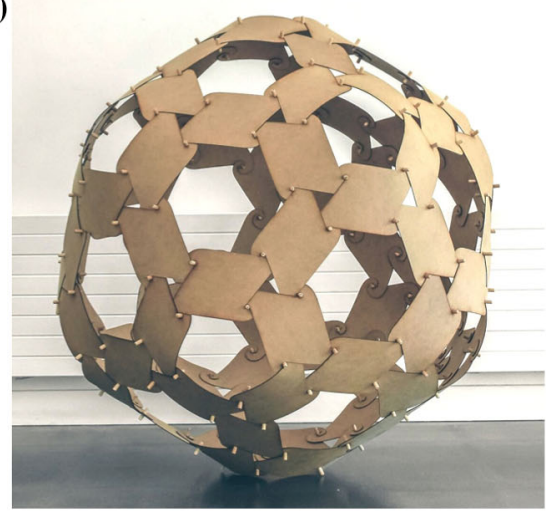

(b)

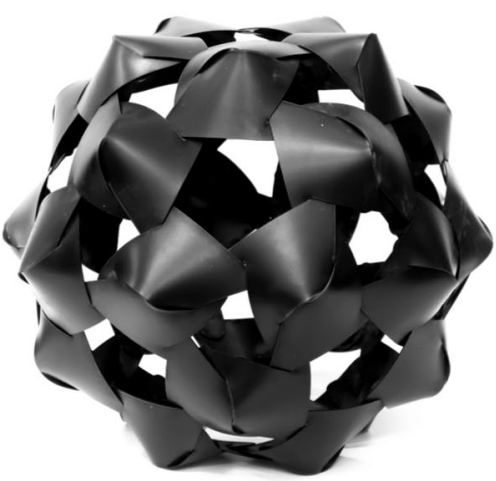

Fig. 12 Two examples of Archimedean solids: a truncated icosahedron with a peg inserted between every two pieces. This technique facilitates assembly, withstands the shear stresses and allows rotation in the joint in order to de-stress the sheets in the areas of greater flexion; $\mathbf{b}$ icosidodecahedron. The assembly of two identical sheets has substituted every sheet from the basic model, doubling the final number of pieces in the structure. This technique provides better curvature and less flexion forces in many polyhedra. Images: authors

This generation study starting from a single piece shows several examples but there are many more than are shown here. The polyhedra can grow by adding pieces and these may also be duplicated (Fig. 12b), triplicated or quadrupled. The cylinders can be skewed, the cones can change their apex, flat patterns can be curved on any developable surface and the width of the tabs may be modified in order to decrease or increase the gaps between pieces.

\section{Further Experiments: Helixes, Quadrics and Russian Dolls}

To complete the experiment in which we have only used the piece shown in Fig. 8e we have developed some complex surfaces, such as helixes (Fig. 13a), quadrics, ellipsoids (Fig. 13b). Russian Dolls (Fig. 13c) are polyhedra that can be nested one inside the other.

The addition of the second dimension in the sheets facilitates the assembly of bending reciprocal structures. The removal of the linear elements and the introduction of only this kind of planar but flexible pieces diminishes many of the problems generated by rigid structural bars. The bending of the sheets reinforces the edges and consequently the linear joint between pieces in a better way than the non-flexible reciprocal pieces do.

\section{Conclusions and Future Works}

The geometry of linear reciprocal structures is highly conditioned by the thickness and curvature of its structural elements. Only some shapes can be constructed by using the chosen elements and pattern. The freedom of design is much greater in 
(a)
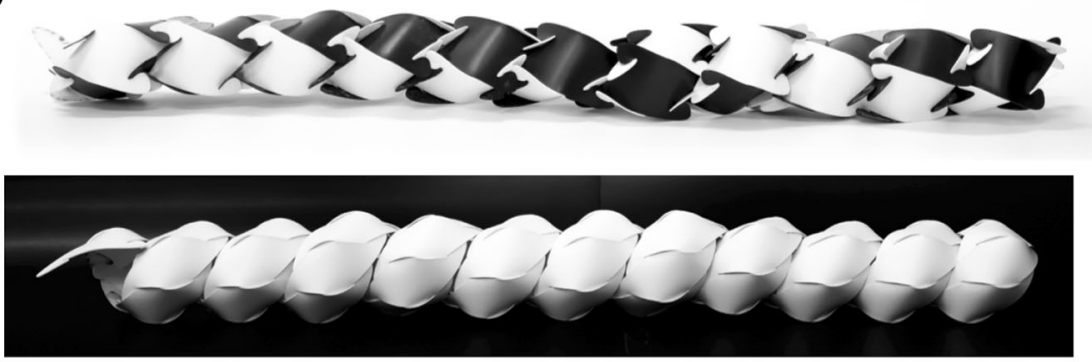

(b)

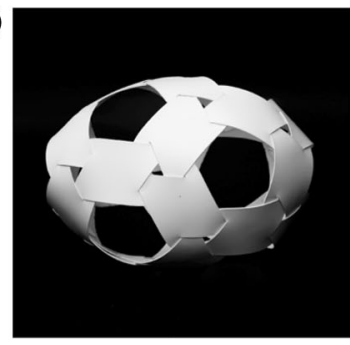

(c)
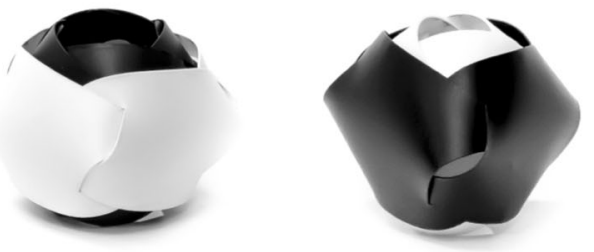

Fig. 13 a Two examples of helixes, $\mathbf{b}$ one example of a quadric surface: an ellipsoid, $\mathbf{c}$ two Russian dolls. Images: authors

laminar reciprocal structures by flexion, since the thickness does not condition the form. When the geometry is complex, the difference between both types of structure is further accentuated and the scope of application is wider: separating panels, light facades, ephemeral constructions... and the architectural possibilities are more interesting.

The generated surface acts as a sheet with perforations. The pieces can be glued to form a unitary element, avoiding shear stresses in the overlaps and reinforcing the joints. Waterproofing, the great problem of reciprocal structures, is more easily achieved than in linear reciprocal structures, as there is more continuity with less protrusion. The shapes generated with simple curvature (cones, cylinders, convolutes...) can have a textile or laminate coating without facings or cuts if the size of the sheet or fabric allows it.

It is important to highlight that there are many types of surfaces that may be covered using this kind of sheet. At this point it is possible to anticipate that any kind of developable surface can be built using sheet patterns. Warped and doublecurvature surfaces may be adapted successfully and the possibilities for further works are really enormous. The application of this type of reciprocal structures on developable surfaces and their adaptation to complex double-curvature forms is explained in the Ph.D. Thesis of Francisco González-Quintial (González 2016). Also interesting is the use of wood sheets and plywood boards either through the structural concept of active form, as presented in the work of Nabaei et al. (2017) or though constructions that take advantage of the potential of the geometric curvature itself without introducing overstresses, as (Narváez-Rodríguez et al. 2015). 

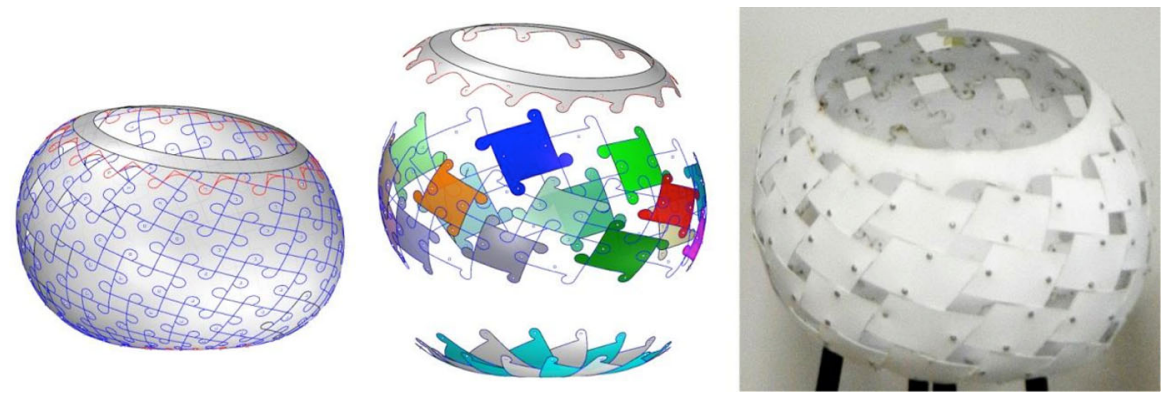

Fig. 14 Laminar reciprocal structure. Construction of a complex shape using different pieces: curvature analysis, mapping and final model. Images: authors

Planar reciprocal structures avoid the complex connections that are generated between the bars organized in reciprocal patterns where solutions of difficult stereotomy appear, although this aspect is minimized with the use of processes of digital manufacture. The techniques of mapping and curvature analysis allow the construction of complex shapes with different pieces, which greatly extends the field of application by adapting the laminar reciprocal structures to new geometries.

In previous sections we have limited our work to a single model of planar piece. The next step consists on the design of different models of sheets able to fit more complex shapes. The production of similar pieces but where each one is slightly distorted and therefore all are different is one of the tasks we are currently developing.

Figure 14 shows the mapping, cutting and construction of a basket using the pattern of Fig. $6 \mathrm{c}$ as generative model. All the pieces assembled look similar, but they are different although they maintain the topological structure of the construction. The pieces have been riveted to give consistency to the object.

This technique is quite easy using planar sheets, which once distorted are able to cover any curved surface. The process of generation-design and fabricationconstruction is nowadays simpler using software like Rhinoceros and laser-cutting machines. Parametric programming helps in the curvature analysis, the adjustment to the complex shape, the design of the sheets and its digital fabrication.

The main goal of our research has been to study and document the transition from reciprocal frame structures into closed reciprocal laminar structures without rigid elements. A future objective is not only to design and construct new models, but also to analyze in depth the behavior of the sheets in these constructions.

The two-dimensional character of the sheets makes them very suitable for the construction of bending reciprocal structures. The elements that compose the structure tend to tighten their overlaps and the stresses at the edges generate a surface with more continuity that typical structures working just under gravity. While the rods forming a structure maintain their rigidity, sheets tend to bend properly if the material has sufficient elasticity. For these reasons, laminar reciprocal structures may be useful in the future of construction because they provide a smart adaption to complex surfaces and could be easily introduced into modern buildings construction techniques. 


\section{References}

Baverel, Olivier and Olga Popovic. 2011. A review of woven structures with focus on reciprocal systems-nexorades. International Journal of Space Structures 26(4): 281-288.

Baverel, Olivier and Alberto Pugnale. 2014. Reciprocal Systems Based on Planar Elements: Morphology and Design Explorations. Nexus Network Journal 16(1): 179-189.

Duvernoy, Sylvie. 2008. An Introduction to Leonardo's Lattices. Nexus Network Journal 10(1): 5-12.

González, Paco. 2016. Adaptación de superficies de doble curvatura mediante superficies desarrollables. EGA: Revista de Expresión Gráfica Arquitectónica 21(27): 210-219.

Kohlhammer, Thomas and Toni Kotnik 2011. Systemic Behaviour of Plane Reciprocal Frame Structures. Structural Engineering International 21 (1): 80-86.

Nabaei, Sina, Olivier Baverel and Yves Weinand. 2017. A hybrid simulation workflow for timber fabric structures. Advancing Wood Architecture: A Computational Approach: 85-93.

Narváez-Rodríguez, Roberto, Andrés Martín-Pastor and Maria Aguilar-Alejandre. 2015. The Caterpillar Gallery: Quadric Surface Theorems, Parametric Design and Digital Fabrication. In: P. Block, J. Knippers, N. Mitra, W. Wang (eds), Advances in Architectural Geometry 2014, 309-322. Springer: Cham.

Parigi, Dario and Alberto Pugnale. 2014. Three-dimensionality in reciprocal structures: concepts and generative rules. Nexus Network Journal 16(1):151-177.

Popovic, Olga. 2007. Reciprocal Frame Architecture. Elsevier Science \& Technology.

Pugnale, Alberto, Dario Parigi, Poul H. Kirkegaard and Mario Sassone. 2011. The principle of structural reciprocity: history, properties and design issues. Proceedings of the IABSE-IASS Symposium 2011: "Taller, Longer, Lighter".

Roelofs, Rinus. 2008. Two- and three-dimensional constructions based on Leonardo grids. Nexus Network Journal 10(1): 17-26.

Sánchez, José, Félix Escrig and Maria-Teresa Rodríguez. 2010. Una aproximación analítica a las mallas recíprocas diseñadas por Leonardo. Informes de la Construcción 62(518): 5-14.

Siza, Alvaro, Eduardo Souto de Moura and Cecil Balmond. 2005. Serpentine Gallery Pavilion 2005. Trolley Books.

Williams, Kim. 2008. Transcription and translation of Codex Atlanticus, fol. 899v. Nexus Network Journal 10(1): 13-16.

Williams, Kim and João Pedro Xavier. 2014. Leonardo 2013 Conference Report: 3-5 June, Perdizes, Portugal. Nexus Network Journal 16(1): 239-246.

Javier Barrallo earned his Ph.D. in Computer Science at the University of Deusto, Bilbao in 1992. He currently belongs to the Department of Applied Mathematics of the UPV-EHU (University of the Basque Country) and teaches in the School of Architecture. He is specialized in the geometry of architecture along history and the relationships between art and science. He works in the field of structural analysis of heritage buildings and the study of complex shapes.

Francisco González-Quintial earned his Ph.D. in Architecture at the University of the Basque Country in 2012 where he currently teaches in the Department of Architecture at the School of Architecture in the UPV-EHU (University of the Basque Country). He is specialized in the geometrical study of buildings and the structural analysis of ancient structures. He also works in parametric programming and the development of complex shapes.

Antonio Sánchez-Parandiet, Research Director, earned his Ph.D. in Architecture at the University of the Basque Country in 2002 where he currently teaches in the Department of Architecture at the School of Architecture in the UPV-EHU (University of the Basque Country). He is specialized in the geometry of surfaces, and works in the study, design and refinement of complex shapes. 